\title{
Sub-30 ms real-time, free-breathing cardiac imaging with SPIRiT
}

\author{
Samuel T Ting ${ }^{1 *}$, Yu Ding ${ }^{1}$, Shivraman Giri ${ }^{2}$, Ning Jin ${ }^{2}$, Orlando P Simonetti ${ }^{1}$, Rizwan Ahmad ${ }^{1}$ \\ From 17th Annual SCMR Scientific Sessions \\ New Orleans, LA, USA. 16-19 January 2014
}

\begin{abstract}
Background
Combined with Variable density Incoherent SpatioTemporal Acquisition (VISTA, [1]), SPIRiT [2] reconstruction can provide an avenue for highly accelerated real-time, free-breathing cardiac imaging. Yet the high computational cost of iterative algorithms limits practical clinical implementation. The Fast Iterative Shrinkage Thresholding Algorithm (FISTA) [3] can potentially reduce the computational cost of SPIRiT [4]. In this work, we combine FISTA optimization with VISTA sampling and spatiotemporally regularized SPIRiT reconstruction to demonstrate the feasibility of real-time cine accelerated to rate 15 , while providing a pathway to practical clinical application.
\end{abstract}

\section{Methods}

Resting cine images in the short axis orientation (48 frames, $224 \times 144$ matrix size, $720 \mathrm{~mm} \times 290 \mathrm{~mm}$ FOV, $8 \mathrm{~mm}$ slice thickness) were acquired (Siemens, Avanto 1.5T, 32 channels) under free-breathing conditions from one healthy volunteer. Data were acquired at acceleration rates ranging from R6 to $\mathrm{R} 15$ (temporal resolutions: $57.6 \mathrm{~ms}$ to $23.0 \mathrm{~ms}$ ) using the VISTA sampling pattern. For faster processing, coil compression from 32 to 12 channels was used, and data were cropped in the frequency encoding direction to 116 pixels. The FISTA optimization algorithm was combined with spatiotemporal L1-regularization within the 3D wavelet domain to take advantage of the VISTA sampling pattern. A GRAPPA reconstruction with a $2 \times 11$ kernel was used as initialization for the FISTA algorithm. Both the $2 \times 11$ GRAPPA kernel and a $7 \times 7$ SPIRiT kernel were estimated from the temporal average of all frames. Acquired

${ }^{1}$ The Ohio State University, Columbus, Ohio, USA

Full list of author information is available at the end of the article data were maintained fixed for the first $10 \%$ of the iterations, after which all data were allowed to be modified by the algorithm. A minimum change in the cost function was used as a stopping criterion, with truncation after 50 iterations. All data were reconstructed offline in MATLAB (version 2013b) using an Intel Core i5 workstation with $16 \mathrm{~Gb}$ memory. SNR of reconstructed images was measured, and images were qualitatively evaluated for aliasing artifact.

\section{Results}

Figure 1 shows reconstructed images at end systole and end diastole at acceleration rates $6,9,12$, and 15. As acceleration increases from R6 to R15, high signal-tonoise is maintained without significant evidence of aliasing artifact. While reconstruction time was just under 20 minutes on a quad-core CPU system, we conservatively expect a tenfold reduction in computation time using GPU processing, allowing us to achieve the speed required for clinically practical application of this approach.

\section{Conclusions}

Combined with VISTA and spatiotemporal regularization, the FISTA implementation of SPIRiT can provide a practical avenue for highly accelerated, real-time, freebreathing cardiac imaging.

\section{Funding}

This work funded by NIH R01 HL102450.

\section{Authors' details}

${ }^{1}$ The Ohio State University, Columbus, Ohio, USA. ${ }^{2}$ Siemens Healthcare, Chicago, Illinois, USA.

Published: 16 January 2014 


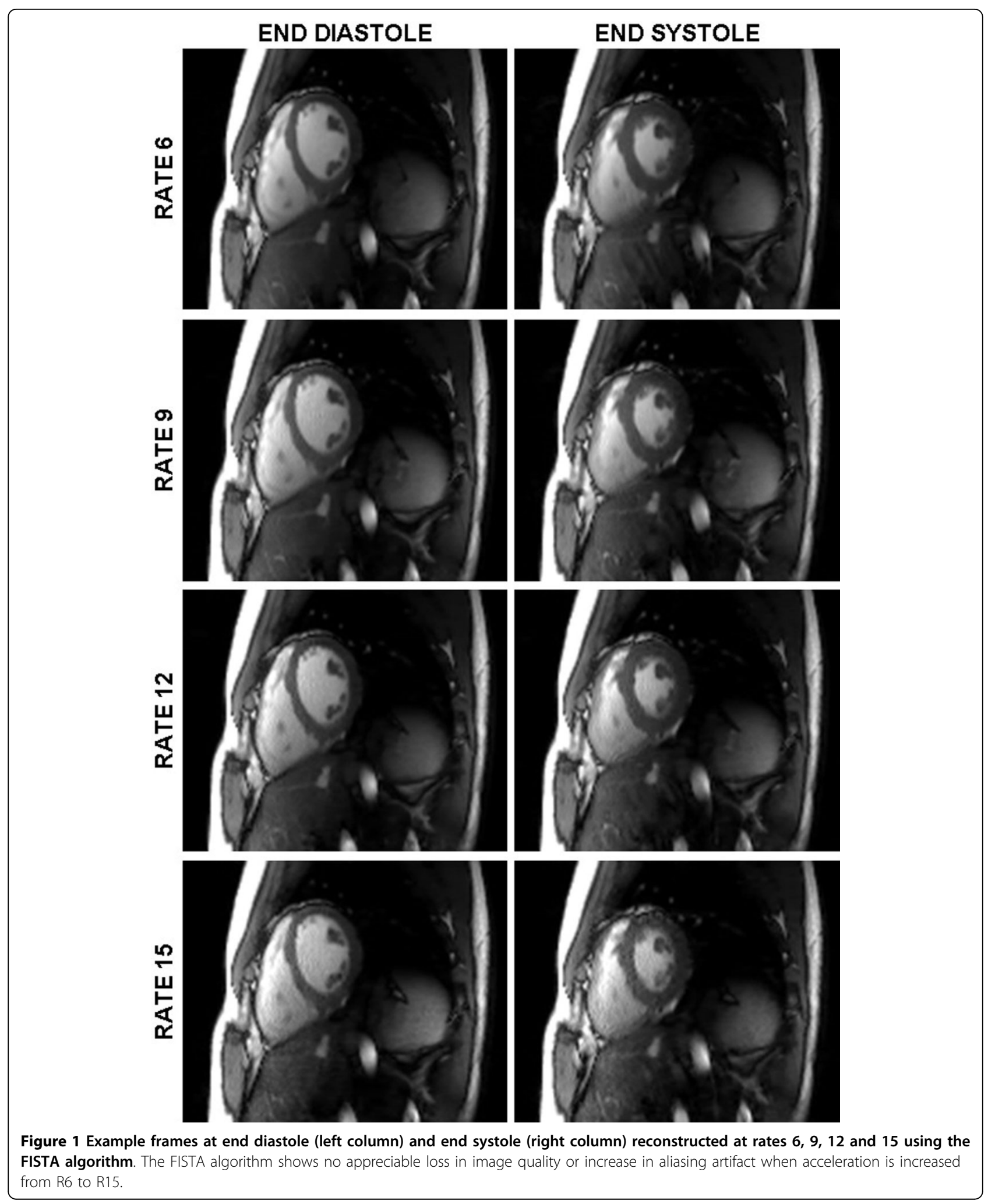




\section{References}

1. Ahmad R, et al: ISMRM DSIR Workshop 2013

2. Lustig M, et al: Mag Res Med 2010, 64(2):457-71.

3. Beck A, et al: SIAM J Imag Sci 2009, 2(1):183-202.

4. Ting ST, et al: Proc ISMRM 2013, 3813.

\section{doi:10.1186/1532-429X-16-S1-W2}

Cite this article as: Ting et al.: Sub-30 ms real-time, free-breathing

cardiac imaging with SPIRiT. Journal of Cardiovascular Magnetic Resonance 2014 16(Suppl 1):W2.

Submit your next manuscript to BioMed Central and take full advantage of:

- Convenient online submission

- Thorough peer review

- No space constraints or color figure charges

- Immediate publication on acceptance

- Inclusion in PubMed, CAS, Scopus and Google Scholar

- Research which is freely available for redistribution 